\title{
COD LIVER OIL AS LOCAL TREATMENT FOR EXTERNAL AFFECTIONS OF THE EYES
}

\author{
BY \\ Edgar STEVenson \\ LIVERPOOL
}

FOR the past six months I have been experimenting with cod liver oil as a local treatment for various eye conditions, and have been able to arrive at certain conclusions, the chief of which is that the oil has an undoubted and rather surprising efficacy as a healing agent. It is obvious that in trying out any new method of treatment the more one can exercise direct supervision of all the cases treated, the better one can assess the results, and it happens that we are favourably placed as regards this supervision, as my partner and I have arranged part of our premises as a private out-patient department, to which are sent eye accidents from the local shipyards and from other works further afield. All of these cases need more skilled attention than can be given by the firm's ambulance departments. We have two skilled assistants and now that shipbuilding is once more becoming active, we deal with a large number of cases. (I started this very interesting sideline over thirty years ago.) In consequence we are able to watch the progress of a case from day to day, or even several times daily if necessary, thus having a great advantage over the ordinary hospital out-patient department.

I am indebted to Dr. Steel of Toxteth Infirmary, Liverpool, for his interesting account in the Lancet of August 10 last, of his treatment of burns with cod liver oil, and was much impressed by the apparent absence of scarring in his cases. I was actually reading his Paper on August 13, when a case most aptly arriveda very severe burn of the inner half of both right lids from a red hot rivet, which adhered to the patient's lids till he had reached ground from the top of the $40 \mathrm{ft}$. ladder on which he was working.

I put in a drop of weak atropine solution, dressed the burn with a thick pad of gamgee soaked in ordinary cod liver oil covered with oiled silk and a bandage, and sent him home and hoped for the best. Next day it seemed to be doing well, no pain, a good deal of mucoid discharge. This was gently wiped off and an oiled pad reapplied. The patient was instructed to get a small bottle of the oil and to keep the pad moist by pouring some oil on the outside of the pad if it seemed to get dry. He was seen daily for a week, by which time the lids were filling up. After that he was allowed to use the oil at home and attend every three days. In exactly four 
weeks the lids were filled up and healed. Both canaliculi had escaped destruction; there was no scarring; skin quite soft, but rather red and itching. He had no treatment except the oil and wiping away any mucus and the preliminary drop of atropine. He returned to work on September 15, and two months later turned up with a localised deep burn of the left eyeball, just clear of the cornea at the inner side. This healed in five days with the oil, leaving a small and rapidly diminishing white patch of scar tissue in the conjunctiva. The right eye looked normal except that the lids had lost their sharp cut edges where burnt, and also, some lashes; no sign of scarring or lacrymation.

This case is typical as regards treatment, and was the most severe of other similar accidents.

One interesting case on December 2, had a splash of liquid caustic (used in cleaning hulls of ships) dropped into his right eye from a height of 30 feet, damaging the conjunctiva of the upper lid, lower half of cornea and conjunctiva, and deeply burning the lower fornix. Very painful, much chemosis and swelling of lids. Oil was used freely and atropine for two days. The eye improved rapidly, but a symblepharon was forming in the lower lid. This was quite soft, very easily separated and did not recur. On December 7 , five days after the accident, upper lid, cornea and conjunctiva were normal except for much redness in the lower fornix. Eye comfortable. Returned to work.

Other corneal conditions.

(1) On February 17, a very deep linear abrasion, about $1 \mathrm{~mm}$. wide, horizontally across $2 / 3$ of cornea, with ragged edges, and a smaller one less deep below it, caused by flying piece off head of rivet. Some particles of dirt were removed from the edges of the wound, drops of weak atropine and oil instilled and pad strapped on. Next day pad removed. Except for an almost invisible white line in cornea, no sign of any injury. The rapid healing of this case surprised us; it really was a very nasty wound.

(2) An old lady, aged 78 years, with dendritic ulcer of left eye. Much pain and photophobia. History of previous attacks, treated by carbolising, and lasting about two weeks. One instillation of oil relieved the pain in ten minutes and stopped the lacrymation. Seen again a week later when except for two small rough spots the ulceration had disappeared and the inflammation and redness had entirely subsided. She had no other treatment.

(3) A child, aged five years, seen at 'Heswall Children's Hospital in October. Under treatment two years for coeliac disease and for about nine months for relapsing keratitis and ulcers of the left eye. Lids much swollen. Under treatment with slight temporary improvement and long periods of relapse. Oil tried as last resort 
three times daily. The ward sister reported the result as "miraculous." Child much improved in twenty-four hours and practically well in four days, but treatment continued for some weeks to clear up nebulae with apparent success. The cornea is now bright and clear except for a very small central nebula. No relapse in four months, but is still having a drop of oil daily.

Hypopyon ulcers show rather curious results. A thick growth of epithelium forms at once (in twenty-four hours) after first instillation, turning the ulcer into an abscess. We find it better not to use the oil till active septic process has subsided. They then seem to heal very satisfactorily with minimum opacity.

I mention these few cases to show that the treatment can be used in a diversity of affections of the eyes. But we have notes of over 150 cases treated at our own clinic and we think that its use has greatly reduced the usual pre-oil number of necessary attendances. I am experimenting at present with the chronic degeneration from mustard gas, and some other chronic conditions, with so far encouraging results, and I want to try it for phlyctenular disease, but have not seen a case of this since I started the treatment.

My partner, Bernard Chavasse, has been using the treatment at the Eye and Ear Infirmary, and reports good results, but he has difficulty in following up the cases. He is specially pleased with the rapid healing of blepharitis and of general soreness of the lids.

To sum up. Cod liver oil applied locally, seems to promote growth of the natural tissues and to inhibit growth of scar tissue, in any case where there is loss of substance. It should be withheld in the early stages of hypopyon ulcer. It is harmless, can be used in conjunction with other necessary treatment, is cheap and easily applied.

The best drop bottle is one made by Messrs. S. T. Pearson and Co., Ltd., Mitcham. It has a vulcanite cap below the rubber which prevents the oil from getting on to the rubber.

The only disadvantage is the smell which, in the small quantities used for the eye, is of no consequence. 